\title{
Hidden addictions, improving eating disorder services and overcoming challenges in autism spectrum disorders
}

\author{
Kevin Glynn ${ }^{1}$ and John Lyne ${ }^{2, *}$ \\ ${ }^{1}$ North Dublin Mental Health Services, Ashlin Centre, Beaumont Road, Dublin 9, Ireland \\ ${ }^{2}$ Royal College of Surgeons in Ireland and North Dublin Mental Health Services, Ashlin Centre, Beaumont Road, Dublin 9, Ireland
}

While society's problems with addiction to illicit substances such as heroin have been well documented, other forms of addiction have remained largely hidden. The advent of instantly accessible and anonymous Internet gambling has led to a significant rise in problem gambling in Ireland. The nature of this problem means it is difficult to study and is often under-reported. In his editorial, O'Gara (pp. 269-271) calls for an immediate enactment of the Gambling Control Bill (2013), pointing out that existing legislation in this area is hopelessly outdated. The new DSM 5 places gambling addiction in the same grouping as alcohol and substance addiction, a reflection of the mounting evidence for the significant morbidity associated with gambling disorders. In their National survey of online gambling behaviours, Columb and O'Gara (pp. 311-319) find that there are many people in Ireland suffering from financial and mental health difficulties due to online gambling. This furthers the case for heightened awareness through public education and regulation of the sector through the enactment of the Gambling Control Bill (2013).

Another often hidden problem in Irish society is addiction to over the counter pain medication. By using in depth interviews, Van Hout et al. (pp. 275-288) offer a fascinating insight into those misusing codeine in Ireland. Themes explored range from the daily routine of codeine abusers, habit formation and negotiating pharmacy sales. Concerning findings include the relative ease of access of codeine-containing medications, as well as a lack of knowledge of the harmful side effects and addictive potential of these medications. Increased education and pharmacovigilance is needed to tackle this increasingly prevalent problem.

Twelve years on from 'A Vision for Change' (2006), it seems that little progress has been made in tackling key issues in the provision of services for eating disorders. In their article, McNicholas et al. (pp. 301-309) explore the perspectives of service providers, service users and the general adult population towards eating disorder

\footnotetext{
* Address for correspondence: John Lyne, Royal College of Surgeons in Ireland and North Dublin Mental Health Services, Ashlin Centre, Beaumont Road, Dublin 9, Ireland.

(Email: johnlyne@mail.com)
}

services in Ireland. What emerges is significant concern from both service providers and parents of service users over the lack of a standardised treatment protocol and clearly defined care pathways for onward referral to specialised services. Another concerning finding is that while dissatisfaction with body image and preoccupation with food remains high among the adolescent population, most are reluctant to report their issues due to the perceived stigma around these issues and a lack of understanding about how to access support services. McDevitt et al. (pp. 289-299) report on a promising study aiming to increase professionals' knowledge and improve services for eating disorders. They found that using a case based interprofessional education approach is an effective way of developing specialist knowledge in this area across disciplines and services.

While our understanding of autism spectrum disorder (ASD) has increased greatly in recent years, there is still relatively little research on those with ASD who find themselves in the Criminal Justice System. In his fascinating perspective piece, O'Sullivan (pp. 333-339) addresses this topic and places it in its historical perspective, while highlighting the need for greater advocacy services and funding for these individuals. Another study by Clarke (pp. 325-328) tries to address further obstacles to treatment among those with ASD by using a novel technology in Autistic patients who are unable to attend a clinic due to anxiety. This shows the potential of telepsychiatry in the treatment of comorbid mental illness among those with ASD.

The use of interviews and qualitative methods in several articles in this issue reminds us that there is much knowledge to be gained from listening directly to service users and service providers with this strategy. Sometimes qualitative studies are the most effective method for exploring complex issues, and such interview techniques remain a valuable way of gaining direct insights into the day to day struggles of those with mental illness.

\section{Conflict of interest}

Kevin Glynn has no conflicts of interest to disclose. John Lyne has no conflicts of interest to disclose. 